\title{
On the orbital period of the cataclysmic variable RZ Leonis
}

\author{
R. E. Mennickent ${ }^{1, \star}$ and C. Tappert ${ }^{1,2}$. \\ 1 Dpto. de Fisica, Facultad de Ciencias Fisicas y Matematicas, Universidad de Concepcion, Casilla 160-C, \\ Concepcion, Chile \\ 2 Dipartimento di Astronomia, Universita di Padova, Vicolo dell'Osservatorio 2, 35122 Padova, Italy \\ e-mail: ctappert@pd.astro.it
}

Received 12 March 2001 / Accepted 4 April 2001

\begin{abstract}
We present a time-resolved study of the Balmer emission lines of RZ Leo. From the analysis of the radial velocities we find an orbital period of $0.07651(26) \mathrm{d}$.
\end{abstract}

Key words. stars: individual: RZ Leonis - stars: novae, cataclysmic variables - stars: fundamental parameters stars: evolution - binaries: general

\section{Introduction}

The cataclysmic variable RZ Leo has been recently confirmed as an SU UMa type dwarf nova after the discovery of superhumps during the December 2000 superoutburst (Ishioka et al. 2000). The photometric period detected in quiescence by Mennickent et al. (1999), $P_{\mathrm{ph}}=0.0756(12) \mathrm{d}$, was also reported during early stages of the superoutburst and thus possibly represents the orbital period of the binary. In this paper we present time resolved spectra of RZ Leo. From the study of the radial velocities of the $\mathrm{H} \alpha$ emission line we found a spectroscopic period of $P_{\mathrm{sp}}=0.07651(26) \mathrm{d}$. We furthermore investigate the variation of the quiescent emission line profile, and find evidence for a prominent emission component from the hot spot region.

\section{Observations and data reduction}

A total of 46 spectra with individual exposure times of 10 min were obtained during two observing runs at La Silla and Las Campanas observatories. The observing schedule and instrumental setup are shown in Table 1. All CCD frames were processed in the standard way, using IRAF (distributed by the National Optical Astronomy Observatories). One-dimensional spectra were extracted, sky-subtracted and wavelength calibrated.

Send offprint requests to: R. E. Mennickent, e-mail: rmennick@stars.cfm.udec.cl

* Based on observations obtained at the European Southern Observatory, ESO proposal 54.E-0812.
Table 1. Journal of Observations. Setup $A$ corresponds to ESO observations with the $2.2 \mathrm{~m}$ telescope using EFOSC2 and grating 4. Setup $B$ corresponds to LCO observations with the $2.5 \mathrm{~m}$ telescope using the Modular Spectrograph and grating 6 .

\begin{tabular}{|c|c|c|c|}
\hline \hline Date (UT) & \multicolumn{1}{c}{ HJD start } & $n_{\text {spec }}$ & setup/resolution \\
\hline $10 / 02 / 95$ & 2449758.7706 & 14 & $\mathrm{~A} / 6 \AA$ \\
$11 / 02 / 95$ & 2449759.7276 & 15 & $\mathrm{~A} / 6 \AA$ \\
$21 / 03 / 95$ & 2449797.7109 & 7 & $\mathrm{~B} / 4.5 \AA$ \\
$23 / 03 / 95$ & 2449799.7113 & 10 & $\mathrm{~B} / 4.5 \AA$ \\
\hline \hline
\end{tabular}

Table 2. Spectroscopic measurements of the averaged spectra. $W_{\lambda}$ is in $\AA, \Delta \lambda, F W Z I$ and $F W H M$ in $\mathrm{km} \mathrm{s}^{-1}$.

\begin{tabular}{|c|c|c|c|c|}
\hline \hline Line & \multicolumn{1}{c}{$W_{\lambda}$} & \multicolumn{1}{c}{$\Delta \lambda$} & \multicolumn{1}{c}{$F W Z I$} & \multicolumn{1}{c}{$F W H M$} \\
$\mathrm{H} \alpha$ & -130 & 600 & 4300 & 2130 \\
$\mathrm{H} \beta$ & -14 & 1150 & 3600 & 2610 \\
$\mathrm{He} \mathrm{I} 5875$ & -34 & 1250 & 3475 & 2170 \\
\hline \hline
\end{tabular}

\section{Spectrophotometric measurements and radial velocity study}

The system was found in quiescence. The spectrum presented here is characterized by double emission lines of $\mathrm{H} \beta$, HeI 5875 and $\mathrm{H} \alpha$ (Fig. 1). In Table 2 we give mean spectroscopic parameters for the main emission lines: equivalent widths, half-peak separation $\Delta \lambda$, full width at half maximum $F W H M$ and full width at zero intensity $F W Z I$.

Radial velocities were measured using a single Gaussian fit to the line profile. These values were analysed 
using both the Scargle and the AOV algorithm (Scargle 1982; Schwarzenberg-Czerny 1989; resp.) implemented in MIDAS. The results in Fig. 2 show several possible frequencies with similar power around a peak frequency of about 13 cycles/d. In order to discriminate between the possible frequencies and derive the true period, we fitted the data with a sine function corresponding to the peak frequency. Then a Monte Carlo simulation was applied in such a way that to each data point a random value from an interval consisting of \pm the sigma of the sine fit was added. The resulting data set was again analysed with the Scargle algorithm, and the highest peak was registered. After a thousand repetitions, the average value within a small interval ( 0.2 cycles/d, determined by visual inspection of the periodogram) around the more recurrent period and its sigma were taken as the resulting period and its error, respectively. The histogram in Fig. 3 shows the resulting distribution for a bin size of 0.01 cycles/d. The derived period was

$P_{\mathrm{sp}}=0.07651(26) \mathrm{d}=1.8363(62) \mathrm{h}$,

which is in excellent agreement with the photometric value $P_{\mathrm{ph}}=0.0756(12) \mathrm{d}$ found by Mennickent et al. (1999). This value places RZ Leo in the midst of the SU UMa period distribution, and near the location of the wellknown systems Z Cha and SU UMa. From the sine fit to the radial velocity data set (Fig. 4), we furthermore obtain a semi-amplitude $K_{1}=91 \pm 12 \mathrm{~km} \mathrm{~s}^{-1}$ and a time $\mathrm{HJD}_{0}=2449759.7489 \pm 0.0022$ corresponding to the superior conjunction of the emitting source (positive to negative crossing of the radial velocity curve). Note that these values were derived by measuring the whole line profile, and therefore cannot be identified a priori with the corresponding parameters of the white dwarf.

The February data set also includes accompanying photometric measurements in the $V$ passband. A nearby comparison star was placed in the slit at the same time as the variable, and differential slit-magnitudes were extracted from the spectra counts. A few $V$ images taken during the night permitted us to roughly calibrate these magnitudes using nearby comparison stars whose standard magnitudes were taken from Mennickent et al. (1999). We estimate that the zero point for the $V$ magnitudes so obtained is accurate to at least 0.1 mag. Results are discussed in the next section.

\section{Discussion}

\subsection{On the orbital and superhump period}

Within the errors, the derived spectroscopic period corresponds well to the photometric period in quiescence, and thus very likely is the orbital period of the binary. A similar period was also detected photometrically during the early stages of the superoutburst. This supports the current understanding of the formation of "early superhumps", which still resemble the orbital period, and which later evolve into "full superhumps" as the accretion

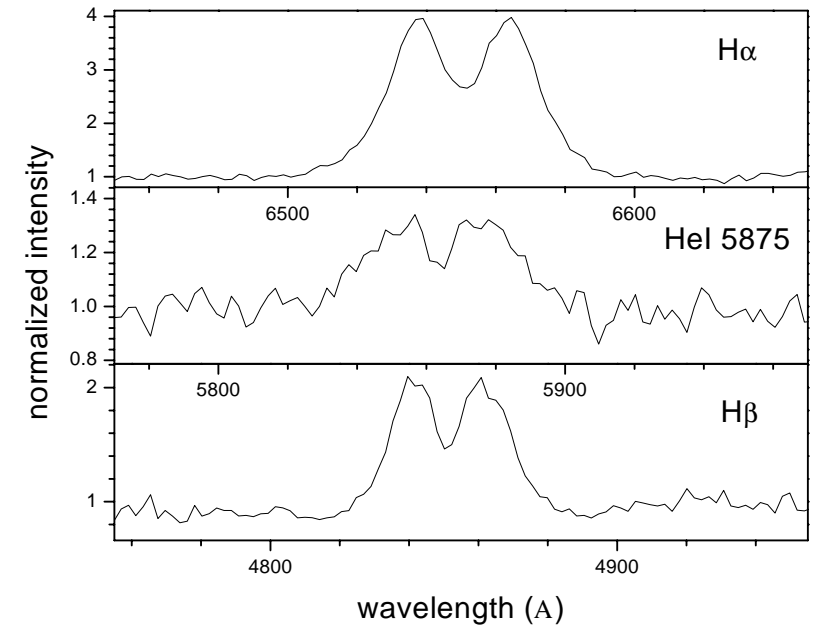

Fig. 1. Averaged emission lines during February 1995.

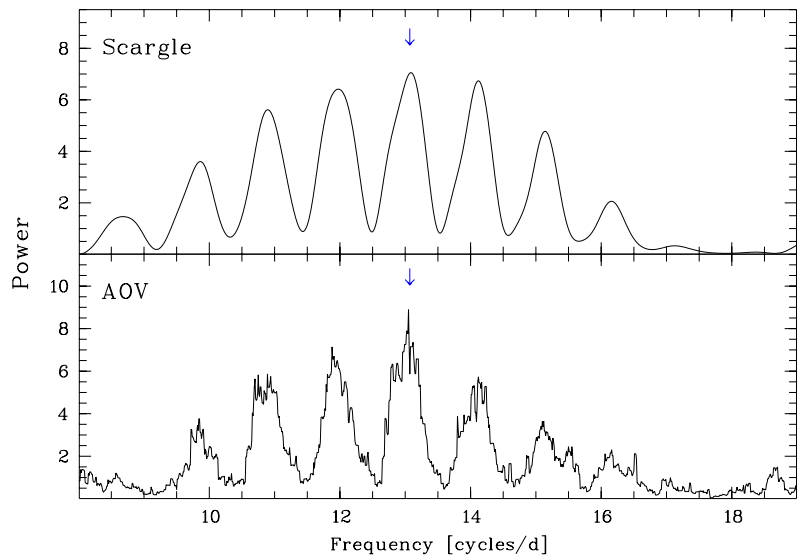

Fig. 2. Scargle (top) and AOV (bottom) periodogram of the radial velocity data. The arrows indicate the frequency corresponding to the derived period.

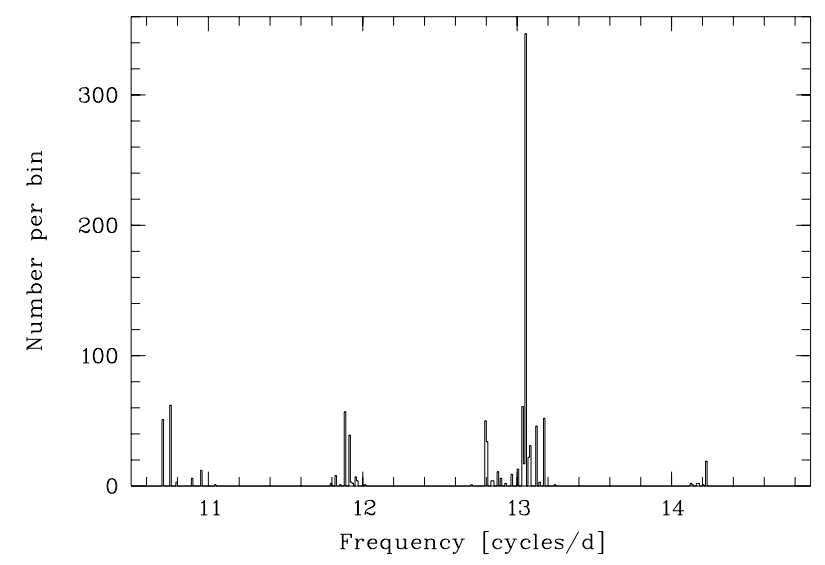

Fig. 3. Histogram of the maximum-peak frequency.

disc is deformed and starts to precess. Considering the superhump period of $0.07857(22) \mathrm{d}$ (Ishioka et al. 2000) we find a period excess between the superhump period $P_{\mathrm{SH}}$ and the orbital period $P_{\text {orb }}$ of

$\epsilon=\frac{P_{\mathrm{SH}}-P_{\mathrm{orb}}}{P_{\mathrm{orb}}}=0.0269(81)$, 


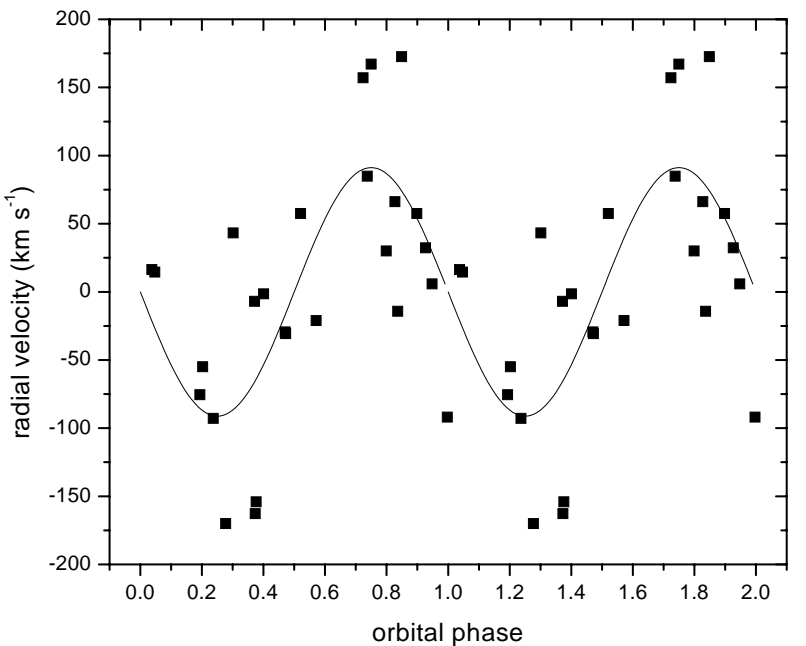

Fig. 4. The $\mathrm{H} \alpha$ emission line radial velocity versus the orbital phase and the best sine fit.

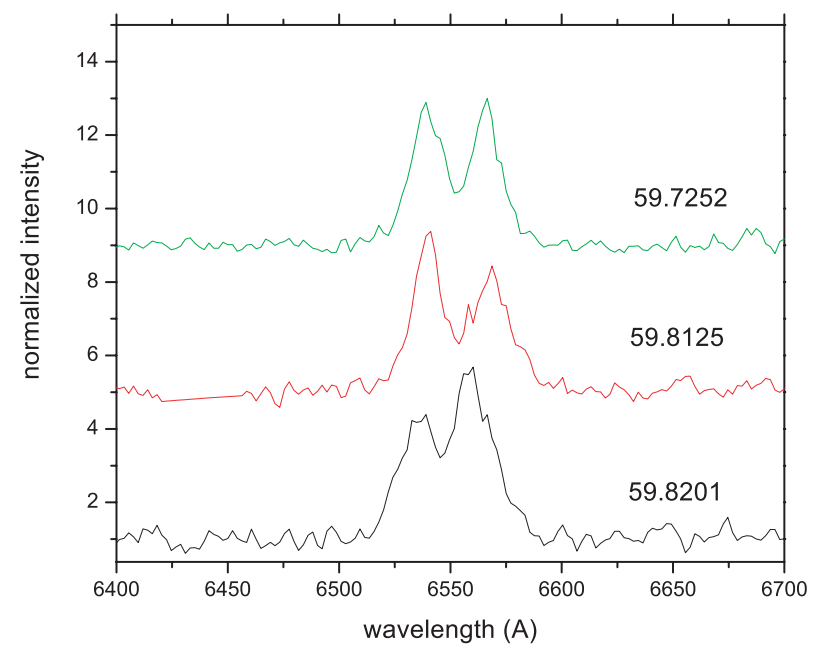

Fig. 5. Selected $\mathrm{H} \alpha$ profiles and their HJD (minus 2449700) showing $V / R$ variations. A vertical spacing of 3 units have been applied to the profiles.

which is a typical value for an SU UMa star of this period (e.g., Patterson 1998).

\subsection{On the changing nature of the hotspot}

As in other well known dwarf novae, variations in the relative strength of the violet and red peak intensity are observed (Fig. 5). These $V / R$ variations could be attributed to the changing aspects of a hotspot in the disk-stream interacting region. This hotspot seems to be also the origin for the variations of the continuum and line strength observed in Fig. 6. The fact that the photometric maximum corresponds to the equivalent width maximum indicates that the hotspot is the source of both continuum and line extra emission. In addition, the displacement of

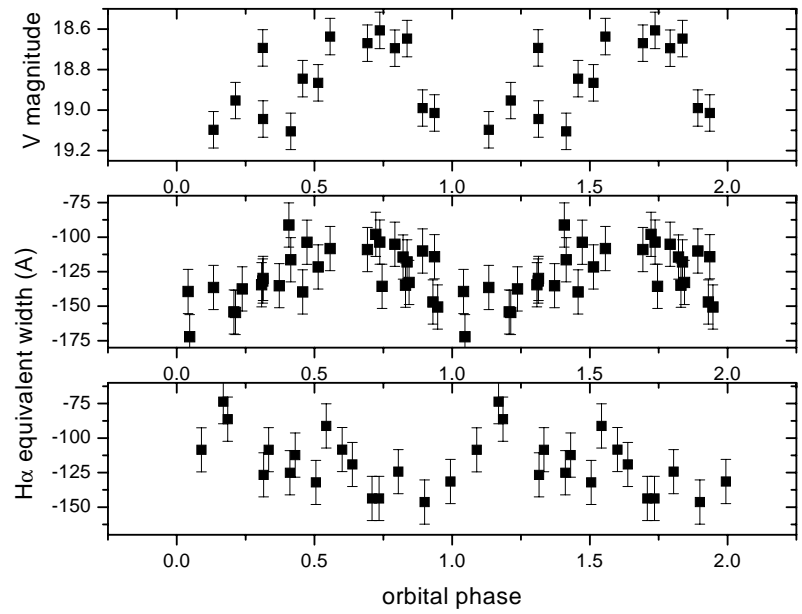

Fig. 6. The $V$ magnitude for the first night of February (upper panel). The $\mathrm{H} \alpha$ emission line equivalent widths for February (middle panel) and March (below panel). A small shift was applied to the equivalent width of February to correct for different nightly mean. Note the non-coherent nature of the equivalent width oscillations.

the maximum phase at different epochs is notable. This could indicate displacements of the hotspot in the binary frame of rest, which was also suggested by Mennickent et al. (1999) to explain the long-term photometric variations. The position of the hotspot is that expected for the stream-disk interacting region in February, but not in March. Anomalous hotspot positions have been also observed by the method of Doppler Tomography and they are not yet fully understood (Szkody 1992).

\section{Conclusions}

1. We have found that the orbital period of RZLeonis is $0.07651(26)$;

2. The hotspot seems to be the source of both continuum and the additional line emission;

3. The position of the hotspot in the system of rest of the binary seems to be variable.

Acknowledgements. This work was supported by Grant Fondecyt 1000324 and DI 99.11.28-1.

\section{References}

Ishioka, R., Uemura, M., Kato, T., Iwamatsu, H., \& Matsumoto, K. 2000, IAUC, 7552

Mennickent, R. E., Sterken, C., Gieren, W., \& Unda, E. 1999, A\&A, 352, 239

Scargle, J. D. 1982, ApJ, 263, 835

Schwarzenberg-Czerny, A. 1989, MNRAS, 241, 153

Szkody, P. 1992, Cataclysmic Variable Stars, ASP Conf. Ser., 29,42

Patterson, J. 1998, PASP, 110, 1132 